\title{
Patient experience of lung volume reduction procedures for emphysema: a qualitative service improvement project
}

\author{
Sara Buttery ${ }^{1}$, Adam Lewis ${ }^{1}$, Inger Oey ${ }^{2}$, Joanne Hargrave ${ }^{2}$, David Waller ${ }^{2}$, \\ Michael Steiner ${ }^{2}$, Pallav L. Shah ${ }^{1}$, Samuel V. Kemp ${ }^{1}$, Simon Jordan ${ }^{1}$ and \\ Nicholas S. Hopkinson ${ }^{1}{ }^{1}$
}

Affiliations: ${ }^{1}$ NIHR Respiratory Disease, Biomedical Research Unit at the Royal Brompton and Harefield NHS Foundation Trust and Imperial College London, London, UK. ${ }^{2}$ Leicester Respiratory Biomedical Research Unit, University Hospitals of Leicester NHS Foundation Trust, Leicester, UK.

Correspondence: Nicholas S. Hopkinson, The Royal Brompton Hospital, Fulham Road, London, SW3 6NP, UK. E-mail: n.hopkinsondic.ac.uk

ABSTRACT The aim of this service improvement project was to gain understanding of the patient experience of lung volume reduction surgery (LVRS) and endobronchial valve (EBV) placement, from referral through to post-discharge care.

Focus group interviews were carried out in two tertiary centres in London and Leicester, UK. Sixteen patients who had undergone lung volume reduction surgery (LVRS), endobronchial valve (EBV) placement, or both, were recruited. Prior to participation in each focus group, participants completed a questionnaire to guide and focus discussion. Thematic analysis identified common themes to the participant experience of receiving lung volume reduction interventions.

Themes included patient focus on declining health and the need to "fight" for a referral; consequences of having procedures and potential unexpected complications; and vulnerability post discharge and limited continuity of care. Participants were clear that the benefits of having had either LVRS or EBV procedures outweighed any difficulties experienced. Participants were keen to have further similar interventions if appropriate.

These data confirm the need to develop more systematic lung volume reduction pathways, provide appropriate information, and ensure that post-discharge care is optimal.

@ERSpublications

Patients feel they have to fight to get a lung volume reduction procedure; a more systematic approach is needed http://ow.ly/82Oy30cLORk

Cite this article as: Buttery S, Lewis A, Oey I, et al. Patient experience of lung volume reduction procedures for emphysema: a qualitative service improvement project. ERJ Open Res 2017; 3: 000312017 [https://doi.org/10.1183/23120541.00031-2017].

This article has supplementary material available from openres.ersjournals.com

Received: March 132017 | Accepted after revision: June 012017

Clinical trial: This study is registered with the National Institute for Health Research with identifier number PB-PG-1014-35051.

Support statement: S. Buttery is funded by The Research for Patient Benefit (RfPB) Scheme of the UK National Institute for Health Research (NIHR) PB-PG-1014-35051. This paper presents independent research funded by the NIHR under its RfPB Programme. The views expressed are those of the authors and not necessarily those of the National Health Service, the NIHR or the Department of Health. Funding information for this article has been deposited with the Crossref Funder Registry.

Conflict of interest: Disclosures can be found alongside this article at openres.ersjournals.com

Copyright $\odot$ ERS 2017. This article is open access and distributed under the terms of the Creative Commons Attribution Non-Commercial Licence 4.0. 


\section{Introduction}

Evidence-based management of chronic obstructive pulmonary disease (COPD) is focused on improving breathlessness, quality of life and healthcare utilisation [1-3]. Inhaled therapies and non-pharmacological treatment options such as pulmonary rehabilitation (PR) provide improvements, but many patients nevertheless experience intractable breathlessness [4]. Interventional approaches such as lung volume reduction surgery (LVRS) may be indicated. LVRS can improve exercise capacity, lung function, quality of life (QoL) and survival in selected patients with emphysema [5, 6]. Evidence is emerging that endobronchial valve placement (EBV) may produce similar benefits [7-12]. Few procedures are performed relative to the disease burden and likely prevalence of suitable individuals [6], and survey data suggest a lack of knowledge among clinicians about indications and processes for referral [13]. We wished to discover more about patient experience of the clinical pathways for lung volume reduction to find ways to improve the process and consider other patient-relevant outcome measures.

\section{Material and methods}

\section{Study subjects}

The 16 patients on interventional procedure databases at the Royal Brompton Hospital ( $\mathrm{RBH}: \mathrm{N}=130)$ or Glenfield Hospital (GH: N=40) who had undergone LVRS, EBV or both between 2008 and 2015 were contacted by telephone and invited to take part in a discussion group exploring their experiences at their local site. At $\mathrm{RBH}$, all patients that attended the focus group who had received valves had done so as part of a clinical trial. Of the patients that attended the focus group at $\mathrm{GH}$, some had received valves as part of clinical trials and some through commissioned pathways. We did not ask individual patients to clarify this on the questionnaire or during the focus group. This was not intended to be an epidemiology study, so we contacted a convenience sample of patients that were on the LVRS and EBV databases at either site and invited them to the focus group. Those who did not answer were not pursued further. A record was not kept of the exact reasons for declining to attend the focus group, but these included a deterioration in the condition, distance from the hospital site, and being uncontactable. Five participants dropped out following an initial expression of interest. These participants failed to attend the focus group. Because the focus groups were undertaken as part of a service improvement project, formal ethics committee approval was not obtained. However, participants provided written, informed consent for their participation.

\section{Study design, methods and analysis}

The 16 participants first completed a questionnaire (supplementary file S1) anonymously. Participant questionnaires explored experiences such as the referral process, the procedure itself and follow-up care (figure 1). The questionnaire was created by N.S.H., S.B. and A.L. to stimulate focused in-depth discussion during semi-structured focus groups led by N.S.H., S.B. and A.L. at both sites. I.O. and J.H. were also present during the focus group in GH. N.S.H. (male) is a consultant physician and reader in respiratory medicine. S.B. (female) is a research physiotherapist and A.L. (male) is a physiotherapist with a $\mathrm{PhD}$ in qualitative research including COPD patients. I.O. is an associate specialist doctor in thoracic surgery. J.H. is a thoracic nurse specialist. N.S.H. and A.L. were employed by Imperial College London and S.B. by Royal Brompton and Harefield NHS Foundation Trust (RBHT). I.O. and J.H. were employed by University Hospitals of Leicester NHS Foundation Trust (UHL). A.L. and S.B. had no previous established relationship with any of the included participants prior to recruitment to the focus groups. N.S.H. had previous involvement with the $\mathrm{RBH}$ patients as their respiratory consultant, and I.O. and J.H. had a clinical relationship with participants in the GH group. We acknowledge that clinical involvement with the research participants may introduce bias, so both S.B. and A.L. asked interview questions at both sites and were the only research members involved in the thematic analysis. Participants were informed about the nature and aims of the research by S.B. on initial telephone contact. Participants then received a written invitation outlining the purpose of the study. N.S.H. introduced the session and explained the rationale. Participants were then given consent forms to sign. Discussions that followed were guided by a semi-structured interview schedule.

The focus group lasted 150 minutes at RBHT and 132 minutes at UHL. Both focus group interviews were digitally recorded, and field notes were made by S.B. during them. Participants were provided with refreshments, and transport costs were reimbursed, but no other financial incentives were offered. Transcripts were not returned to participants for comment. S.B. and A.L. coded the transcripts independently, coding was recorded using Microsoft Excel, with the coding tree of the RBHT focus group attached as an appendix (supplementary file S1). Themes were derived from the data according to the thematic analysis method presented by Braun and Clarke [14]. Participant checking of the themes was performed with a representative participant from the RBHT and UHL focus groups. The COREQ (Consolidated Criteria for Reporting Qualitative Research) checklist for qualitative research was followed in reporting the data. 


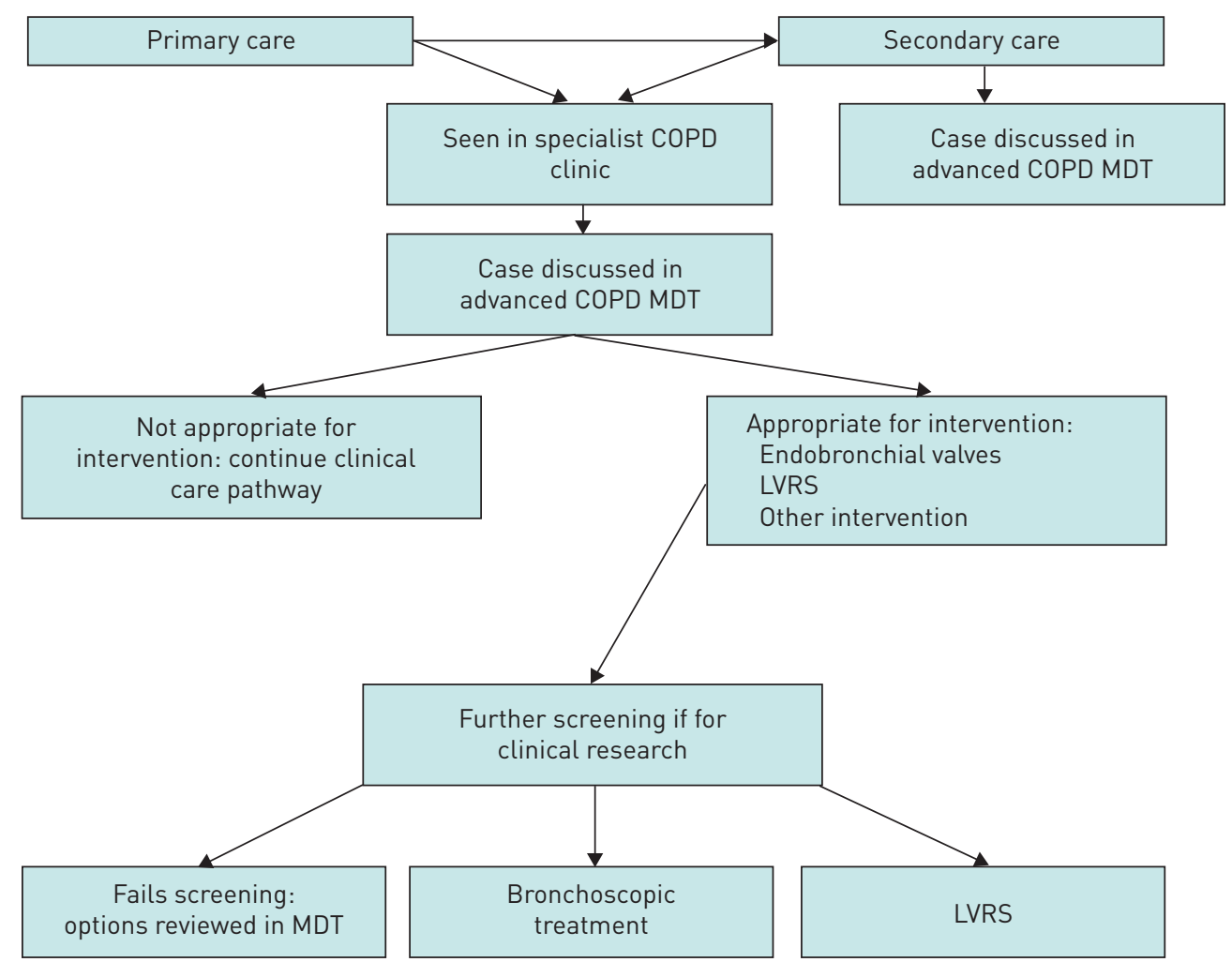

FIGURE 1 Pathway for patients being referred for lung volume reduction. COPD: chronic obstructive pulmonary disease; MDT: multidisciplinary team; LVRS: lung volume reduction surgery.

\section{Results}

The mean (SD) age of participants in the focus groups was 63 (8) years, and there were equal numbers of men and women (table 1). Three participants had undergone EBV and 13 LVRS as a first procedure. Seven participants had undergone a second procedure (1=EBV, 6=LVRS). Participants described having developed significant limitation in their daily living activities by the age of 55 (10.5) years, with an average of 9 (7.5) years between first being diagnosed and developing significant day-to-day limitation. On average, patients had undergone their first intervention 3.8 (2.2) years prior to participation in the focus groups. Participants reported 14.3 (8.9) days in hospital post LVRS and 1.5 (1.0) days post EBV. Patients included in the focus group were largely a cohort of patients that received EBVs where the procedure was carried out as a day case. Current best-practice guidelines now recommend that patients remain in hospital for three nights post procedure.

\section{TABLE 1 Demographic data of patients attending focus groups at the Royal Brompton and}

\section{Glenfield Hospitals}

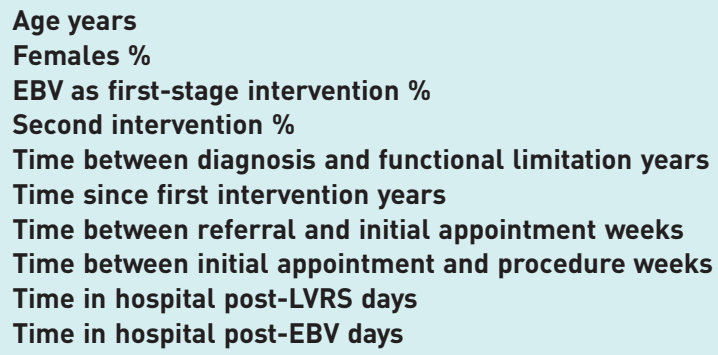


Patients completed a visual analogue scale (VAS) to describe the effect of the procedure they had undergone. Participants felt that lung volume reduction (LVR) improved their global health (mean (SD) of $+2.53(2.61)$ on a -8 to $+8 \mathrm{VAS}$ ). Posed as a Yes/No question, 14 out of 16 participants would have the procedure again.

Interviews were transcribed verbatim and coded independently by S.B. and A.L. Thematic analysis exposed the following themes: 1) patient focus on declining health; 2) consequences of having the operation; 3) vulnerability and limited continuity of care post discharge.

\section{Patient focus on declining health}

Participants blamed themselves for their deterioration. Participants felt like they were in a fight to earn their chance to have an operation. They discussed this fight according to their level of deterioration, their perceived future worsening of their condition, and negotiation with healthcare professionals:

[Participant 1, RBH] "If you want it, you gotta earn it."

[Participant 2, RBH] "You've got to earn your right. I'm telling you. It really does, I mean if you don't fight for it...”

Participants had often found out about potential interventions themselves, with few being referred directly by their general practitioner (GP):

[Participant 1, UHL] “A lot of GPs though, they don't do anything about it at all. Mine didn't.”

Participants understood that this may be due to lack of "specialist" knowledge about novel procedures held by generalists.

A timely referral to secondary and tertiary care to a specialist COPD clinic is likely to be beneficial for this population. According to questionnaire responses, there had been an average of 28.5 (64.6) weeks to see a consultant to discuss lung volume reduction (LVR) following initial referral from primary care. From then it took an average of 38.9 (34.7) weeks to receive intervention.

\section{Consequences of having the operation}

Participants' inpatient experiences were generally positive, but they suggested that communication could be improved. Many had chest infections or other post-operative complications for which they felt unprepared:

[Participant 3, RBH] "I had the massive surgical emphysema... I was blind for two days."

[Participant 1, RBH] "I got the surgical emphysema again but not so severe this time."

[Participant 2, UHL] "I don't think I realised until I couldn't see. My eyes all closed up and my daughter came in and screamed."

One individual was able to reflect on the difference between LVRS and EBV because she had received both procedures. She felt instantly better following EBV insertion, compared to a longer recovery with LVRS, although she had benefited from both:

[Participant 1, UHL] "When you go in you can hardly breathe, and the next day when you've had it it's like a weight has been lifted off of you and you can actually walk and not have to stop!"

\section{Vulnerability and limited continuity of care post discharge}

Participants who required oxygen in the post-procedure period felt they were not prepared for this and this could be addressed prior to the procedure:

[Participant 4, RBH] “Today, just going $100 \mathrm{~m}$, I'm having to use oxygen. So I'm going another way where it seems to be getting worse."

[Participant 1, UHL] "I got home and there was this great big machine there and that did me straight away and I thought that is it, I've had it, I'm going to die. I really did. And I hadn't worried until then.”

The second quote highlights the perceived impact having oxygen at home can have on people with lung disease [15]. Without adequate pre-procedure discussion, patients may feel that a need to use oxygen at home means they are in or nearing a terminal stage of their condition. Patients should be given information about the likelihood and consequences of requiring oxygen post procedure, and for how long they may need to use it.

Some participants described a lack of continuity of care and feeling vulnerable as a result:

[Participant 2, RBH] "My own GP I never saw. Nobody came to see me.”

[Participant 1, RBH] “At home, I didn't get a nurse come to see me, and that took forever." 
Participants discussed feeling vulnerable at home once they had left hospital after receiving a high level of support on the wards. Having access to a Respiratory Early Discharge Service (REDS) improved the post-discharge experience:

[Participant 3, UHL] "If you wanted to talk to a nurse they were always there you can talk to them without them having to come out."

Furthermore, many participants stressed the importance of a follow-up appointment with their surgical team post procedure and felt that being routinely offered PR after the procedure would be beneficial:

[Participant 5, RBH] "I haven't been back since I had the operation."

It is recommended that all patients due to receive interventional procedures should have completed PR beforehand. It is not known whether receiving PR as part of a post-procedure standard of care improves outcomes from the interventions, although this is plausible.

Despite some negative experiences, most participants would agree to further surgery or other interventions if these options were available to them:

[Participant 6, RBH] “Can we get a lung transplant?”

[Participant 2, RBH] "I want my right lung done now."

Those who had second LVRS/EBV procedures also reported positive experiences:

[Participant 3, UHL] "I've been so good if I slip back it'll take me longer to... So I had the second one done. I’ve never looked back."

A comprehensive account of a thematic analysis audit trail and patient responses is available online (supplementary file S2).

\section{Discussion}

The main themes identified around undergoing a lung volume reduction procedure were as follows: 1) participants often felt that they had had to "fight" for a referral prior to receiving their intervention; 2) although participants felt that interventions had been beneficial overall, they had experienced complications, some of which had been unexpected; 3) post discharge, participants experienced vulnerability and limited continuity of care in the absence of a specialist discharge service.

\section{Significance of findings}

These findings are from a service improvement project at two established centres in the UK; however, they offer generalisable suggestions for how the patient experience of LVR procedures could be improved, from referral through to follow-up post discharge. Delayed referral is typical, and many patients described a long pathway to treatment and often had to initiate referrals themselves. A survey of British Thoracic Society members revealed significant overestimation of the risks associated with LVRS, uncertainty about indications, and an absence of multidisciplinary team (MDT) structures to support assessment [13]. A specific MDT LVR pathway has been suggested to give healthcare professionals access to the indications for LVR procedures [16]. An approach incorporating systematic consideration for LVR in all patients who have an Medical Research Council dyspnoea score of 4 or 5 at the end of pulmonary rehabilitation may be appropriate. At this point, individuals' condition will have been optimised and consideration of LVR should not be delayed. Further research is needed to develop assessment metrics for COPD healthcare teams (both in primary and secondary care settings) to identify patients with significant hyperinflation and burden of breathlessness who would be potential candidates for LVR using routine data. Many individuals with severe COPD being managed in Primary Care may be appropriate for interventional procedures but are not being referred for specialist review. Other patients may be referred but only after months or years of 'fighting' to receive such an intervention and based on their own investigations. Over time as their disease worsens they may progress beyond the safety criteria for intervention even if they have the "right" pattern of emphysema.

UK National Institute for Health and Care Excellence technology guidance allows for the placement of EBVs subject to funding being agreed by the appropriate Clinical Commissioning Group. Other patients may only have access to such interventions by entering trials with limited recruitment numbers and deadlines. The latter was probably the case when the participants included in the focus groups were being considered for procedures, which may explain some of the delay. Access to LVR should be considered in quality standards of care when managing COPD patients [17, 18].

It is important to ensure that patients are informed of possible complications, as these can be distressing. Expected potential complications post intervention include pneumothorax, which occurs in approximately $20-30 \%$ of patients, and a similar proportion develop acute bronchitis, pneumonia or exacerbate [19]. Valve 
migration may also occur, but this is rarer [19]. Out of the 16 participants interviewed, four reported surgical emphysema and one of these had surgical emphysema after both LVR operations. This complication can be extremely frightening, and the information provided to patients should mention it specifically.

Pulmonary rehabilitation is recommended following acute exacerbations of COPD and may also be a useful intervention to enhance recovery, improving exercise capacity and quality of life following surgery. The development of the COPD discharge care bundle highlighted the importance that patients have access to clear post-discharge support $[20,21]$. The involvement of a REDS service was seen as valuable for participants in this study. Issues with post-operative care are not unique to thoracic surgery, as follow-up is increasingly moved into primary care [22].

Individuals in the focus group were keen on having further procedures should they become available to them. This is evidence to support that LVRS and EBV interventions are both tolerable and often deemed successful from the patient experience. It may be that certain individuals may also benefit from psychology input prior to receiving interventional procedures to ensure that each participant fully comprehends the impact of agreeing to have the procedure. Access to psychological services may also help in the recovery process post procedure. Standardised consent documentation for each interventional procedure should include information regarding possible requirement for oxygen therapy post procedure and complications such as surgical emphysema. These potential complications may need to be discussed with patients repeatedly to ensure that they are understood.

At present there are no data comparing the effectiveness of EBV placement and LVRS directly, and genuine equipoise regarding long-term outcomes following either valve placement or LVRS. The CELEB trial (ISRCTN19684749), funded by the UK National Institute for Health Research (NIHR), will provide further data on differences by randomising suitable patients to one or other treatment and evaluating the response at 12 months.

\section{Methodological issues}

The focus groups included individuals who had undergone a procedure, so we cannot comment on the clinical pathway experience of those who had been assessed but found to be unsuitable. In addition, those with worse outcomes or bad experiences might have chosen not to participate or, alternatively, may have been more vocal. To address this, the questionnaire explicitly asked for both bad and good experiences; however, responder bias is still possible.

A potential source of bias was the participation of some members of the treatment team in the focus groups. To minimise this bias, A.L. and S.B. were included in the team as individuals with no prior clinical involvement with participants. Questioning during focus groups was shared among S.B., A.L. and N.S.H. Despite the presence of members of the treatment team, we did not find that participants were deterred from making negative comments. Indeed, it enabled them to feedback directly to service leads. Furthermore, as this was a service improvement project it was important that the leads of each service were present. N.S.H., I.O. and J.H. were not involved in the initial stages of thematic analysis.

The LVRS sites in this study have different post-treatment pathways. Due to the locality of patients receiving interventions in GH, a REDS is provided for patients in GH. This is not possible in RBH. There may be shared learning to develop other pathways nationally, for example the introduction of post-intervention pulmonary rehabilitation. The generalisability of the findings may be put into context with other units with similar service provision.

Only four people across the sites received EBV, and data saturation could not be confirmed with further interviews or focus groups due to the novel nature of the procedure. We performed member checking with a participant of the focus group at each site in order to confirm that quotes and themes discussed in this paper were accurate according to the patients' experience: "I have no real comments to make, on the contents, it seems a very detailed and accurate report." This participant from RBH reported that he continues to experience benefit to his quality of life by continuing to go on walking holidays and work full time in a demanding job. The feedback from the UHL participant was similar, in that the themes reflected the experience of both LVRS and EBV patients and he gave a favourable opinion about his intervention and care.

The information on time spent going through the assessment process was based on patient recall rather than medical records, so it may not be accurate.

\section{Conclusions}

The participants in the groups were clear that the benefits of having either LVRS or EBV procedures outweighed any difficulties they experienced, and they were keen to have further similar interventions if beneficial. This information may help clinicians who may not refer patients because of overestimating the 
risk versus benefit of intervention. Work is needed to improve referral pathways, patient information and post-discharge care.

\section{Acknowledgements}

This work was supported by the NIHR Respiratory Biomedical Research Unit at Royal Brompton and Harefield NHS Foundation Trust and Imperial College London and the National Institute for Health Research Leicester Respiratory Biomedical Research Unit. The views expressed are those of the author(s) and not necessarily those of the NHS, the NIHR or the Department of Health.

N.S.H. and S.B. conceived the study. N.S.H., S.B.., A.L., I.O. and J.H. took part in the focus groups. S.B. and A.L. performed thematic analysis. S.B. produced a first draft of the manuscript, and all authors contributed to interpretation of the findings and approved the final draft. N.S.H. is the guarantor.

\section{References}

1 GOLD. Global Strategy for the Diagnosis, Prevention and Management of Chronic Obstructive Pulmonary Disease. http://goldcopd.org/download/326/

2 Zoumot Z, Jordan S, Hopkinson NS. Emphysema: time to say farewell to therapeutic nihilism. Thorax 2014; 69: 973-975.

3 Hopkinson NS, Baxter N. Breathing SPACE - a practical approach to the breathless patient. NPJ Prim Care Respir Med 2017; 27: 5.

4 Kelly JL, Bamsey O, Smith C, et al. Health status assessment in routine clinical practice: the chronic obstructive pulmonary disease assessment test score in outpatients. Respiration 2012; 84: 193-199.

5 Criner GJ, Cordova F, Sternberg AL, et al. The National Emphysema Treatment Trial (NETT): Part II: Lessons learned about lung volume reduction surgery. Am J Respir Crit Care Med 2011; 184: 881-893.

6 Clark SJ, Zoumot Z, Bamsey O, et al. Surgical approaches for lung volume reduction in emphysema. Clin Med (Lond) 2014; 14: 122-127.

7 Davey C, Zoumot Z, Jordan S, et al. Bronchoscopic lung volume reduction with endobronchial valves for patients with heterogeneous emphysema and intact interlobar fissures (the BeLieVeR-HIFi study): a randomised controlled trial. Lancet 2015; 386: 1066-1073.

8 Garner J, Kemp SV, Toma TP, et al. Survival after endobronchial valve placement for emphysema: a 10-year follow-up study. Am J Respir Crit Care Med 2016; 194: 519-521.

9 Hopkinson NS, Kemp SV, Toma TP, et al. Atelectasis and survival after bronchoscopic lung volume reduction for COPD. Eur Respir J 2011; 37: 1346-1351.

10 Klooster K, ten Hacken NHT, Hartman JE, et al. Endobronchial valves for emphysema without interlobar collateral ventilation. $N$ Engl J Med 2015; 373: 2325-2335.

11 Zoumot Z, LoMauro A, Aliverti A, et al. Lung volume reduction in emphysema improves chest wall asynchrony. Chest 2015; 148: 185-195.

12 Faisal A, Zoumot Z, Shah PL, et al. Effective bronchoscopic lung volume reduction accelerates exercise oxygen uptake kinetics in emphysema. Chest 2016; 149: 435-446.

13 McNulty W, Jordan S, Hopkinson NS. Attitudes and access to lung volume reduction surgery for COPD: a survey by the British Thoracic Society. BMJ Open Respir Res 2014; 1: e000023.

14 Braun V, Clarke V. Using thematic analysis in psychology. Qual Res Psychol 2006; 3: 77-101.

15 Kelly CA, Maden M. How do respiratory patients perceive oxygen therapy? A critical interpretative synthesis of the literature. Chron Respir Dis 2014; 11: 209-228.

16 Rathinam S, Oey I, Steiner M, et al. The role of the emphysema multidisciplinary team in a successful lung volume reduction surgery programme. Eur J Cardiothorac Surg 2014; 46: 1021-1026.

17 NICE. Chronic Obstructive Pulmonary Disease Quality Standards. London, National Institute for Health and Care Excellence, 2011

18 RightCare. NHS Atlas of Variation in Healthcare for People with Respiratory Disease: Reducing Unwarranted Variation to Increase Value and Improve Quality. London, NHS, 2012.

19 Slebos DJ, Shah PL, Herth FJF, et al. Endobronchial valves for endoscopic lung volume reduction: best practice recommendations from expert panel on endoscopic lung volume reduction. Respiration 2017; 93: 138-150.

20 Hopkinson NS, Englebretsen C, Cooley N, et al. Designing and implementing a COPD discharge care bundle. Thorax 2012; 67: 90-92.

21 Laverty AA, Elkin SL, Watt HC, et al. Impact of a COPD discharge care bundle on readmissions following admission with acute exacerbation: interrupted time series analysis. PLOS ONE 2015; 10: e0116187.

22 Berg K, Årestedt K, Kjellgren K. Postoperative recovery from the perspective of day surgery patients: a phenomenographic study. Int J Nurs Stud 2013; 50: 1630-1638. 